\title{
Post-vaccination rabies sero-survey in Georgia, 2015
}

\author{
Natia Kartskhia*, Lena Ninidze, Lasha Avaliani and Tengiz Chaligava
}

National Food Agency of the Ministry of Agriculture, Tbilisi, Georgia

\section{Objective}

The objective of this survey was to study vaccination coverage and quality in dogs in Georgia through the detection of post-vaccination antibodies.

\section{Introduction}

Rabies is endemic in Georgia with up to 100 confirmed cases in animals per year. There is an estimated 350,000 domestic and stray dogs/cats in Georgia. The prophylactic vaccination of domestic animals against rabies was reestablished in Georgia in 2013. Each year since 2013, coverage has increased aiming to cover approximately $70 \%$ of the total population of dogs/cats in Georgia.

\section{Methods}

Only vaccinated dog populations were included in the serosurvey. Using random selection, five locations were selected. The survey was conducted over a period of 4-8 weeks after vaccination. In order to study vaccination coverage, the total dog population was registered. Samples were taken only from vaccinated dogs (confirmed by vaccination papers) and samples were sent to the Laboratory of the Ministry of Agriculture where they were tested for the presence of antibodies using ELISA. Epidemiological information and GPS coordinates were recorded in the electronic integrated disease surveillance system (EIDSS) and geographic information system (GIS).

\section{Results}

Out of 572 dogs in sampled villages, 373 animal's vaccination was confirmed leading to $65 \%$ vaccination coverage. Out of 255 samples, 241 were suitable for testing; 237 samples $(98.3 \%)$ were positive for the existence of antibodies. Antibody titer was not measured.

\section{Conclusions}

Based on the results of the survey, it can be seen that vaccination coverage is generally not high $(65 \%)$ and needs improvement. The vaccination quality (as determined through the existence of antibodies) is good (98.3\%). In further surveys, antibody titers must be measured in order to extract more information regarding vaccination quality.

\section{Keywords}

Rabies; Vaccination; Sero-survey

\section{Acknowledgments}

The research study described in this presentation was made possible by financial support provided by the US Defense Threat Reduction Agency. The findings, opinions and views expressed herein belong to the authors and do not reflect an official position of the Department of the Army, Department of Defense, or the US Government, or any other organization listed 